\title{
Bemerkungen zum Profil Hörmating/Obb.
}

\author{
Von KARL Brunnacker, München \\ Mit 1 Abbildung im Text
}

\begin{abstract}
$\mathrm{Z}$ us a mmenfassung: Das von E. Ebers (1960) beschriebene und gedeutete Profil von Hörmating wird einer kritischen Betrachtung unterzogen und neu interpretiert. Demnach kommt hier nur ein an eine einzige Landoberfläche gebundener Boden, typologisch als stark entwickelte Parabraunerde vor, wie sie seitens der Paläopedologie im gesamten mitteleuropäischen Raum als Auswirkung interglazialer Verwitterung angesehen wird. Im Bereich der nicht mehr aufgeschlossenen Folge mit Seekreide und Torf ist zumindest die Deutung der hangenden Feinsedimente als Seeton fraglich; denn über dem aufgeschlossenen Bodenrest liegt eine Fließserde (Abb. 1), wie sie entsprechend dem Profil Hörlkofen - die periglaziale Sedimentationsfolge der Würmeiszeit einleitet. - Das wichtigste offene Problem in der Gliederung des Jungpleistozäns liegt weiterhin in der Frage einer Verknüpfung des mittels vegetationskundlicher Untersuchungen rekonstruierten K.limaablaufes des Riß/Würm-Interglazials einschließlich der beginnenden Würmeiszeit mit dem Beginn des Periglazialgeschehens der Würmeiszeit.
\end{abstract}

A bstract: A critical review and a new interpretation is given of the Pleistocene deposits at Hörmating described by E. EbERs (1960) There is only a single „Parabraunerde“ horizon, of the type considered by all authorities as evidence of interglacial weathering in Central Europe. In the upper part of the section (today covered with debris) E. EBERs described lacustrine mud and peat. There, at least the interpretation of the fine-grained sediments as lacustrine deposits is questionable, since a horizon with solifluction (fig. 1) lies upon the soil horizon mentioned above; and usually that is the base of the Würm periglacial deposits, as can be seen, for instance, in the Hörlkofen section. Consequently, the most important problem in Younger Pleistocene stratigraphy is still open: how to connect the latest traces of the climatic evolution of the Riß/Würm interglacial period, as reconstructed on paleobotanical evidence, with the earliest periglacial deposits of the Würm glacial period.

Eines der wichtigsten Ergebnisse des Löß-Symposiums, das anläßlich des 6. INQUAKongresses 1961 in Lublin veranstaltet wurde, waren die gleichartigen Feststellungen über die Gliederung der würmeiszeitlichen Periglazialprofile in den verschiedenen Lößprovinzen Mitteleuropas auf der Grundlage der Paläopedologie (Vorträge von: I. LIEBEвотн, V. LožEK, J. Fink, K. Brunnacker). Demnach ist u. a. die Göttweiger Bodenbildung als Ausdruck eines „großen Würminterstadials“ hinfällig. Auch anwesende Anhänger eines derartigen Göttweig-Interstadials vermochten hiergegen keine ernsthaften bzw. überhaupt keine Einwände vorzubringen. Als Ersatz oder Untermauerung für diesen je nach Auffassung nie vorhandenen oder nun verlorengegangenen bzw. problematisch gewordenen locus typicus scheint das von E. EBERs (1960) beschriebene, von H. Gross (1960) zusätzlich parallelisierte und von E. KRAUS (1961) noch einmal behandelte Profil von Hörmating geradezu prädestiniert. Dieses Hörmatinger Profil habe ich im Jahre 1961 mehrfach besucht; einmal zusammen mit Frau Dr. E. EBers. Nachdem Frau Dr. EBERS trotz aller Einwände weiterhin die ursprüngliche Auffassung aufrecht erhält (1961), sind einige kritische Bemerkungen zu diesem Profil angebracht.

Das Profil zeigte im Jahre 1961 den in Abb. 1 dargestellten, seitlich durch Angaben von E. EBErs (1960) ergänzten Aufbau:

1. Mit Sicherheit läßt sich nur der Boden einer einzigen Landoberfläche erkennen und zwar in ehemaliger Oberhanglage als einwandfreier B-Horizont einer interglazialen Parabraunerde und in ehemaliger Unterhang- und Hangfußlage als die dazugehörige standortsbedingte Modifikation. Zwischen den beiden fossil erhalten gebliebenen Bodenresten ist die ehemalige Landoberfläche, die somit die Form eines flachen Rückens besaß, etwas geköpft. Der hier ursprünglich ebenfalls vorhandene Boden wurde z. T. solifluktiv abgetragen. Dieses Abtragsmaterial überdeckt als Fließerde den noch am Hangfuß in situ 


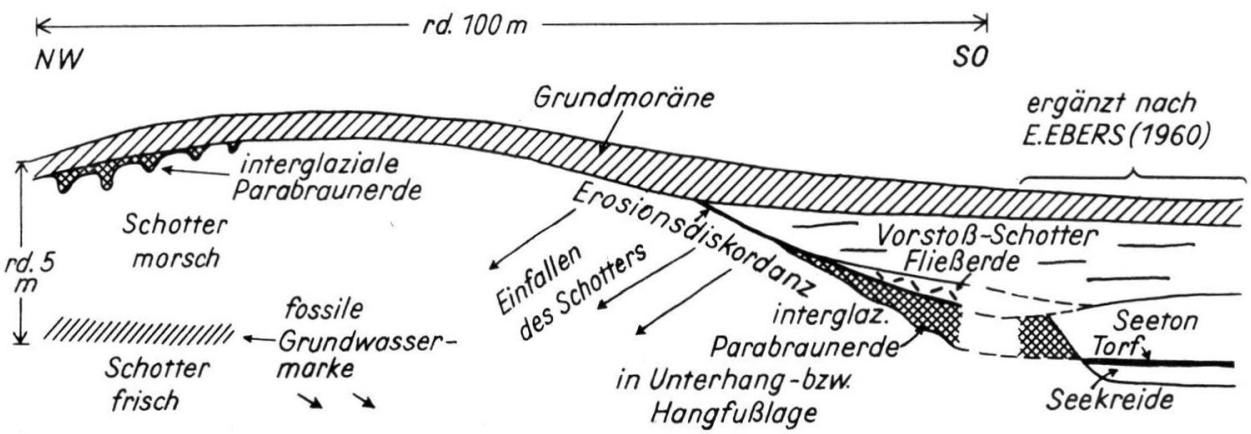

Abb. 1. Der Aufbau des Profils von Hörmating nach meinen Beobachtungen im Jahre 1961, seitlich nach den Angaben von E. EBERs (1960) ergänzt.

befindlichen Bodenrest. Die Seitenerosion bei Ablagerung des darüber folgenden Schotters wird ebenfalls ausräumend gewirkt haben. Vor allem aber wurde der Boden im Bereich des ehedem vorhandenen Rückens durch den darüber hinweggehenden Gletscher aufgearbeitet. Es liegt somit eine Erosionsdiskordanz zwischen einem unteren Schotter und einem oberen Schotter bzw. Grundmoräne. Auch die Abb. 4 bei E. Kraus (1961) bedarf entsprechender Korrektur.

2. Richtige Beobachtung des bei meinen Besuchen nicht mehr aufgeschlossenen Kontaktes zwischen diesem fossilen Boden und der Seekreide vorausgesetzt (E. EBERs 1960, Abb. 2), bleibt es unverständlich, weshalb diesem Boden interstadialer Charakter bei einer doch auch für Laien auf dem Gebiet der Paläopedologie bemerkenswerten Mächtigkeit von mehr als $2 \mathrm{~m}$ zugesprochen wurde (die heutigen in jeder Hinsicht vergleichbaren Böden dieses Gebietes zeigen eine Mächtigkeit von max. $1 \mathrm{~m}$ ). Es läge doch nahe, folgendermaßen zu gliedern: 1. Boden = Riß/Würm-Interglazial, 2. Ausräumung einer Rinne im Bereich der Reliefdepression = „WI“, 3. Füllung der Depression bzw. Rinne mit Seekreide und Torf $=$ "Göttweig-Interstadial“. Das Hauptmotiv, weshalb nicht zu dieser Lösung gegriffen wurde, scheint das „frische“ Aussehen dieses tieferen Teiles des Schotters zu sein. Abgesehen davon, daß dies kein stratigraphisches Kriterium ist, zeigen die Gerölle (besonders aus Dolomit und Flysch) im oberen Abschnitt dieses unteren Schotters durchaus die Anwitterungserscheinungen, wie sie in älteren Ablagerungen häufiger vorzukommen pflegen.

3. Eine gewichtige Rolle spielen weiter die $C^{14}$-Daten aus dem die Seekreide überlagernden Torfhorizont (Torf: $45300 \pm 1000$, Holz: über $53000 \mathrm{C}^{14}$-Jahre). Aus solchen unterschiedlichen $\mathrm{C}^{14}$-Daten ungleichwertigen Materials, noch dazu bei so weit zeitlich zurückliegender Ablagerung, Altersunterschiede zwischen Holz und Torf abzuleiten, ist wohl möglich, jedoch keinesfalls zwingend; denn Holz liefert bessere Werte als Torf, wie Torf wiederum bessere zu liefern vermag als z. B. Humus. Darauf hat übrigens H. Gross (1957, S. 149) selbst hingewiesen, was ihn aber nicht hinderte (1960), hier ebenfalls die Torfdatierung zur Grundlage einer Stratigraphie zu machen. Wer das älter datierte Holz als aus wesentlich älteren Ablagerungen aufgearbeitet und eingedriftet deuten will, kann dies nicht auf Grund der unterschiedlichen $\mathrm{C}^{14}-\mathrm{Daten}$ tun, sondern muß andere, stichhaltigere Gründe anführen. Hier wurde also wieder einmal die sicherlich sehr wertvolle C ${ }^{14}-\mathrm{Me}-$ thode in ähnlicher Weise überfordert, wie der Elefantenzahn von Münsingen (vgl. K. D. AdAM 1960), dem E. Ebers (1955) in einem vergleichbaren Zusammenhang ebenfalls einige Bedeutung beimißt.

Die Humuszonen im Profil von Unterwisternitz zeigen übrigens ein Cl4-Alter von mehr als 55000 Jahren und die von Oberfellabrunn sind dementsprechend zu korrigieren (vgl. B. KuIMA \& J. KUKLA 1961). 
4. Über dem Torf soll ein „Seeton“ mit Lößkomponente liegen. In den bei meinen Besuchen noch offenen Teilen der Kiesgrube war über dem fossilen Boden zum Teil eine hellbraune Fließerde als Gemisch aus A3- und B-Horizontmaterial der verlagerten Parabraunerde aufgeschlossen (Abb. 1). Diese an Restgeröllen reiche Lage weist einen verhältnismäßig hohen Schluffgehalt auf, der von einer Lößkomponente (E. SCHÖNHALs nach E. EBERS 1960), aber genau so gut von in den Schottern vorhandenem und dann verwittertem Flyschmaterial abgeleitet werden kann. Doch ist dies letzten Endes unwesentlich. Von Interesse ist hingegen der (mündliche) Hinweis von Frau Dr. EBers, daß der hangende Seeton, abgesehen von grauer Färbung, gleichartige Ausbildung zeigt. Nun macht. aber graue Färbung noch lange nicht aus einer Fließerde einen Seeton. Es könnte vielmehr an synsedimentäre wie postsedimentäre Vergleyung dieser Fließerde gedacht werden, wobei ein Zusammenhang zwischen den die Reduktion des Eisens auslösenden, im Sediment zirkulierenden Grundwässern und dem unterlagernden Torf nicht von der Hand zu weisen ist. Auf die sonstigen nicht immer ganz verständlichen Deutungsversuche, wie vor allem die „Verwitterung “ einer hangenden „Seekreide“ zu diesem „Seeton“, näher einzugehen, erübrigt sich.

Das Prinzip der würmeiszeitlichen Abfolge des Profils von Hörmating ist damit bis zu einem gewissen Grad dem ähnlich, das von Hörlkofen beschrieben wurde (K. BrunNACKER 1956): Mit einer Basisfließerde wird die würmeiszeitliche Periglazialfolge eingeleitet, darüber kommt im Glazialbereich ein Vorstoßschotter und dann die Grundmoräne, im Periglazialbereich hingegen die weiter differenzierbare würmeiszeitliche Lößabfolge.

Die Probleme des Profils von Hörmating liegen in einem ganz anderen Bereich, als von E. Ebers (1960), H. Gross (1960) und E. Kraus (1961) dargestellt - nämlich in der Beziehung zwischen dem sicher interglazialen Boden und der "Seekreide“-Torf-Folge, die gemäß der Abb. 2 von E. EBERs (1960) durch eine Erosionsphase voneinander getrennt sein müssen. Daran würden sich interessante Folgerungen anschließen lassen, wenn dieser Kontakt in der dargestellten Weise wirklich eindeutig beobachtet worden wäre; doch ist dies, wie Frau Dr. EBERs bei der gemeinsamen Besichtigung zu erkennen gab, keineswegs der Fall.

Nicht nur im Alpenvorland bestehen Schwierigkeiten, die pollenanalytisch untersuchten Profile des Letzten Interglazials mit den vorwiegend paläopedologisch untersuchten würmeiszeitlichen periglazialen Profilen zu verknüpfen. Daß hier noch Probleme offen sind, wurde schon vor Jahren betont (K. Brunnacker, z. B. 1957). Dazu wird Hörmating jedoch kaum einen Beitrag zu liefern vermögen.

Ergänzend sei noch auf sonstige „Nachweise“ des Murnauer bzw. Göttweig-Interstadials in Bayern eingegangen. Den fossilen „Murnauer Boden“ im Sinne von E. Kraus (1955) vermochte ich trotz des empfohlenen Schürfens nicht zu finden. Das einzige eventuell diskutable Profil innerhalb der von mir besuchten Aufschlüsse könnte in der Kiesgrube am nördlichen Ortsausgang von Riegsee liegen. Doch handelt es sich dabei (falls nicht rezent) gegebenenfalls um einen interglazialen Bodenrest einer Parabraunerde, nicht um einen interstadialen.

Das von H. Gross (1959, S. 71) zitierte angebliche "Göttweig-Vorkommen“ in der Ziegelei Korbacher in Kitzingen beruht auf einem Mißverständnis seitens Herrn Gross; darauf wies Herr Schönhals bei einem erneuten Besuch des Profils hin. Hinsichtlich der Funde am Unterislinger Weg in Regensburg wird H. Gross (1959) seine Auffassung wohl ebenfalls revidieren müssen; denn die genaue Situation geht eindeutig aus der Originalliteratur hervor. Das Problem am Profil von Ried bei Neuburg a. d. Donau ist nicht durch "minutiöse" feinstratigraphische (H. Gross 1959), ansonsten am Kern der Sache vorbeigehende und durch die Paläopedologie überholte Methoden zu lösen (z. B. E. W. Guenther 1961). 
Zur Methodik sei noch ergänzend bemerkt: Eine Untersuchung der Lößprofile allein vom Sediment her ist nicht geeignet, eine stratigraphische Einstufung zu erreichen. Eine Untersuchung mit dem Schwergewicht auf paläopedologischen Kriterien bringt hingegen zumindest vergleichbare Deutungen. So war es anfänglich meist nicht immer ganz einfach, die Profile des Alpenvorlandes mit denen von Böhmen in Übereinstimmung zu bringen. Heute jedoch, wo gleiche Arbeitsmethoden unter Betonung paläopedologischer Kriterien Anwendung finden, stellen sich vergleichbare Ergebnisse ein, so daß eine Parallelisierung unschwer möglich ist.

Außerdem ist der Fundhorizont des Faustkeils von Ried nicht gesichert. Dieses Artefakt mag wohl dem tieferen Abschnitt des Profils entnommen sein, es könnte aber - abgesehen von Erwägungen, die sich hier nicht weiter erörtern lassen - vielleicht doch auch der gerade an Lehmwänden mit Baggereibetrieb recht kräftig ausgebildeten Verzugszone entnommen sein.

\section{Schriften}

AdAM, K. D.: Das Mammut aus dem Grabental bei Münsingen (Kanton Bern). Ein überfordertes Leitfossil. - Ecl. geol. Helvetiae 53, S. 481-491, Basel 1960.

Brunnacker, K.: Das Lößprofil von Hörlkofen/Obb. - N. Jb. Geol. Paläontol., Mh. 1956, S. 424433, Stuttgart 1956. - - Die Geschichte der Böden im jüngeren Pleistozän im Bayern. Geol. Bavarica 34, München 1957.

Ebers, E.: Hauptwürm, Spätwürm, Frühwürm und die Frage der älteren Würmschotter. - Diese Zeitschr. 6, S. 96-109, Ơhringen 1955. - - Drumlinkerne, ältere Würmschotter und das Würm-Interstadial-Profil von Hörmating/Obb. - Ebendort 11, S. 64-76, Ơhringen 1960. - - The new found Laufen-Interstadial in Upper Bavaria. - INQUA, 6. Kongress, Abstracts of Papers, S. 21-22, Łodz 1961.

Gross, H.: Die Fortschritte der Radiokarbon-Methode 1952-1956. - Diese Ztschr. 8, S. 141-180, Óhringen 1957. - - Noch einmal: Riß oder Würm? - Ebendort 10, S. 65-76, Ơhringen 1959.

Guenther, E. W.: Sedimentpetrographische Untersuchung von Lössen. I. Teil. - Fundamenta, Reihe B, 1, Köln, Graz 1961.

Klima, B. \& KukLA, J.: Absolute chronological Date of Czechoslovak Pleistocene. - Inst. Geolog. Prace, 34, S. 171-174, Warzawa 1961.

Kraus, E.: Zur Zweigliederung der südbayerischen Würmeiszeit durch eine Innerwürm-Verwitterungsperiode. - Diese Ztschr. 6, S. 75-95, Öhringen 1955. - - Die beiden interstadialen Würmböden in Südbayern. - Ebendort 12, S. 43-59, Öhringen 1961.

Manuskr. eingeg. 13. 2. 1962

Anschrift des Verf.: Privatdozent Dr. K. Brunnacker, München 27, Möhlstraße 12a. 